\title{
Norovirus; the principal threat of food borne illness world wide: a review
}

\begin{abstract}
Gastroenteritis, that was probably due to norovirus, was first described by Zahorsky in 1929 as 'winter vomiting disease'. However the agent was not identified until 1972, when virus particles were first visualised by electron microscopy (EM) in faeces obtained from an outbreak. The outbreak had occurred in 1968 at a school in Norwalk, Ohio, US, with a high attack rate of illness among students and teachers. The illness was characterised by nausea, vomiting and diarrhoea with duration of illness of 12-24hours. The discovery of the virus through EM was important because this was the first virus detected that was specifically associated with cases of acute gastroenteritis. For decades the role of the virus as a causative agent has been hampered by the insensitivity of microbiological diagnostics. Previously it could not be grown in cell culture and there was no small animal model. The only alternative was to test on human volunteers. To overcome this gap, investigators at Baylor College of Medicine engineered human intestinal tissue from stem cells isolated from the small intestine. When the researchers infected the cultured gut tissue with different strains of norovirus, some virus strains grew well in the cells while others did not. To promote virus growth, the team then tried adding bile from humans and other animals, including cows and pigs, to the intestinal cells. Researchers found that bile was required for replication of some virus strains and enhanced the growth of other strains in the cells. The authors say this new cultivation method could have applications for food safety and the development of new diagnostics, vaccines and therapeutics for norovirus. ${ }^{1}$
\end{abstract}

Keywords: norovirus, outbreak, research, cultivation, electron microscopy
Volume 5 Issue 2 - 2017

\author{
Pratikchhya Adhikari \\ Department of Microbiology, St. Xavier College, Nepal
}

Correspondence: Pratikchhya Adhikari, Department of Microbiology, St. Xavier College, Maitighar, Kathmandu, Nepal, Tel 9845196965, Email pratif52@gmail.com

Received: May 17, 2017 | Published: August 03, 2017

\section{Introduction}

Norovirus (previously called "Norwalk-like virus" or NLV) is a member of the family Caliciviridae. ${ }^{2,3}$ The name derives from the Latin for chalice-calyx-meaning cup-like, and refers to the indentations of the virus surface. ${ }^{3}$ The family of Caliciviridae consists of several distinct groups of viruses that were first named after the places where outbreaks occurred. ${ }^{4}$ The first of these outbreaks occurred in 1968 among schoolchildren in Norwalk, Ohio. ${ }^{5}$

The prototype strain was identified four years later, in 1972, and was the first virus identified that specifically caused gastroenteritis in humans. ${ }^{5,3}$ Other discoveries followed, with each strain name based on the location of its discovery-e.g., Montgomery County, Snow Mountain, Mexico, Hawaii, Parmatta, Taunton, and Toronto viruses. ${ }^{2,6}$ A study published in 1977 found that the Toronto virus was the second most common cause of gastroenteritis in children. ${ }^{7}$ Eventually this confusing nomenclature was resolved, first in favor of calling each of the strains a Norwalk-like virus, and then simply, a norovirus - the term used today. 5,3

\section{Transmission}

Norovirus causes nearly $60 \%$ of all foodborne illness outbreaks. Norovirus is transmitted primarily through the fecal-oral route, with fewer than 100 norovirus particles needed to cause infection., ${ }^{2,3}$ Transmission occurs either person-to-person or through contamination of food or water. ${ }^{2,3,8} \mathrm{CDC}$ statistics show that food is the most common vehicle of transmission for noroviruses; of 232 outbreaks of norovirus between July 1997 and June 2000, 57\% were foodborne, 16\% were spread from person-to-person, and 3\% were waterborne. ${ }^{9,10}$ When food is the vehicle of transmission, contamination occurs most often through a food handler improperly handling a food directly before it is eaten. Infected individuals shed the virus in large numbers in their vomit and stool, shedding the highest amount of viral particles while they are ill. ${ }^{3}$ Aerosolized vomit has also been implicated as a mode of norovirus transmission. ${ }^{4}$ Previously, it was thought that viral shedding ceased approximately 100 hours after infection; however, some individuals continue to shed norovirus long after they have recovered from it, in some cases up to 28 days after experiencing symptoms. ${ }^{10,11}$ Viral shedding can also precede symptoms, which occurs in approximately $30 \%$ of cases. ${ }^{5}$ Often, an infected food handler may not even show symptoms. ${ }^{9}$ In these cases, people can carry the same viral load as those who do experience symptoms. ${ }^{3,5,9}$

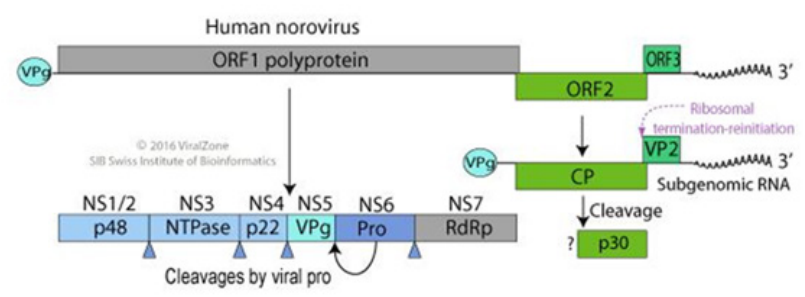

\section{Structure and genome}

The virion is non-enveloped, capsid of about $38-40 \mathrm{~nm}$ in diameter, with $\mathrm{T}=3$ icosahedral symmetry. The capsid is composed of $180 \mathrm{VP} 1$ proteins. Small empty virions are about $23 \mathrm{~nm}$ in diameter, and would be of $\mathrm{T}=1$ icosahedral symmetry, composed of $60 \mathrm{VP} 1$ proteins. The genome is Monopartite, linear ssRNA positive sense of 7.3 to $8.3 \mathrm{~kb}$. The 5'-terminus is linked to a VPg protein and the 3'-terminus has a poly(A) tract.n (VPg)is covalently linked to genome, whereas 3'-terminus is polyadenylated. The virion RNA is infectious and 
serves as both genome and viral messenger RNA. Cleavage of ORF1 polyprotein by the virus-encoded 3C-like cysteine proteinase yields the mature nonstructural proteins. Subgenomic RNA encodes the capsid protein and VP2 minor structural protein which is expressed through RNA termination-reinitiation. Murine norovirus express an alternative ORF from the CP mRNA by leaky scanning. ${ }^{12}$

\section{Epidemiology}

Intensive research in the last years has thus led to that Norovirus today is recognized as the most common cause of acute gastroenteritis in humans. ${ }^{13,14}$ The Center for Disease Control and Prevention in the United States reported that Norovirus account for over $96 \%$ of all foodborne viral gastroenteritis, and over 23 million annual infections in the United States alone. ${ }^{15}$ It has furthermore been estimated that each year, it causes 64,000 episodes of diarrhea requiring hospitalizations, and 900,000 clinic visits among children in industrialized countries. Moreover, Norovirus is estimated to cause up to 200,000 deaths of children less than 5years of age in developing countries and other studies have indicated that this virus cause up to half of all cases of gastroenteritis world-wide, resulting in the striking number of over 267 million annual infections. Molecular epidemiology of Noroviruses was detected in Nepalese children with acute diarrhea between 2005 and 2011 showing increase and predominance of minor genotype GII.13. ${ }^{13}$ These facts highlight the impact of Norovirus in the society, both in industrialized and developing countries, with no vaccine, effective treatments, or prevention strategies currently. ${ }^{16}$

\section{Period of shedding and infectivity}

Previous human volunteer studies have found that viral shedding in stools coincided with onset of illness and did not extend more than 72 hours after the onset of the first symptom. However, viral RNA has been detected using molecular techniques for up to three weeks after onset of illness. Studies have shown prolonged viral shedding and duration of illness due to norovirus infection, and demonstrated that excretion of virus occurred after cessation of symptoms and in infected individuals with no clinical symptoms. A study in Victoria examined clinical symptoms and norovirus excretion among elderly residents during an outbreak of gastroenteritis at an ACF. The study found that acute symptoms lasted 3-4days and the median excretion time for norovirus was 8.6days. Viral excretion was not related to clinical symptoms or the appearance of stools. Similarly, a study of the natural history of calicivirus in the Netherlands found that norovirus occurred in all age groups, clinical symptoms lasted a median of 5 days and stool samples were positive by PCR for norovirus up to three weeks after the of onset of illness Prolonged viral shedding, either symptomatic or asymptomatic, has implications for transmission. While asymptomatic food handlers may be important in spreading disease, the significance of viral excretion in the absence of symptoms is unclear. Marshall et al. suggest that excretion of norovirus by people who are asymptomatic may act as a significant potential infectious reservoir in the community. Viral shedding is greatest during the acute illness and the amount of virus excreted decreases rapidly with recovery. Viral shedding in stools is greatest over the first 24-48hours. There is no evidence that infected food handlers and health care workers should be excluded from the workplace for longer than 48 hours after cessation of symptoms. ${ }^{17}$

\section{Diagnostic methods}

Researchers have succeeded in culturing norovirus in human intestinal cells. But this has not be implemented in action till date. Diagnostic methods focus on detecting viral RNA or antigen. Most public health and clinical virology laboratories can test for norovirus using real-time reverse transcription-polymerase chain reaction (RTqPCR) assays.

\section{RT-qPCR assays}

RT-qPCR assays are the preferred laboratory method for detecting norovirus. These assays are very sensitive and can detect as few as 10 to 100 norovirus copies per reaction. They use different primers to differentiate genogroup I and genogroup II norovirus. RT-qPCR assays are also quantitative and can provide estimates of viral load. The assays may be used to detect norovirus in stool, vomitus, foods, water, and environmental specimens.

\section{Conventional RT-PCR assays for genotyping}

Conventional RT-PCR followed by sequence analysis of the RTPCR products is used for norovirus genotyping. Typically, a partial region of the capsid gene, such as region $\mathrm{D}$, is used by laboratories participating in CaliciNet, a national laboratory surveillance network for norovirus outbreaks coordinated by CDC.

\section{Enzyme immunoassays}

Rapid commercial assays, such as enzyme immunoassays (EIAs), that detect norovirus antigen have recently been developed. However, these kits have poor sensitivity $(50 \%)$ and are not recommended for diagnosing norovirus infection in sporadic cases of gastroenteritis. The RIDASCREEN Norovirus 3rd Generation EIA was recently cleared by Food and Drug Administration for preliminary identification of norovirus when testing multiple specimens during outbreaks. However, samples that test negative should be confirmed by a second technique, such as RT-qPCR. Thus, EIA kits should not replace molecular methods during outbreak investigations. ${ }^{1,9}$

\section{Treatment}

Noroviruses, like other viruses, don't respond to antibiotics, which are designed to kill bacteria. No antiviral drug can treat noroviruses, but in healthy people the illness should go away on its own within a couple of days. Most people don't have any long-term problems from the virus.

To prevent dehydration, plenty of liquids, especially water and juices should be taken. Children should be given an oral rehydration solution (such as Pedialyte) to replace lost fluids and electrolytes. Sugary drinks must be avoided, which can make diarrhea worse, as well as alcohol and caffeinated beverages, which could lead to dehydrate further. Symptoms of dehydration include dizziness when standing, dry mouth, and a decrease in urination. Severe dehydration is sometimes treated with intravenous (IV) fluids. ${ }^{3,9}$

\section{Acknowledgements}

None.

\section{Conflict of interest}

The author declares no conflict of interest.

\section{References}

1. News-Medical.net. Researchers develop new method to culture norovirus in intestinal cells. An AZoNetwork Site. 2016. 
2. Glass RI, Jacqueline Noel, Tamie Ando, et al. The Epidemiology of Enteric Caliciviruses from Humans: A Reassessment Using New Diagnostics. Journal of Infectious Diseases. 2000;181(Suppl 2):S254 S261.

3. Treanor JJ, Dolin Raphael. Norwalk Virus and Other Calciviruses. In: Mandell D, Bennett's, editors. Principles and practice of infectious diseases. 5th ed. 2000. p. 1949-1956.

4. Said MA, Perl TM, Sears CL. Healthcare epidemiology: gastrointestinal flu: norovirus in health care and long-term care facilities. Clin Infect Dis. 2008;47(9):1202-1208.

5. Glass RI, Parashar UD, Estes MK. Norovirus Gastroenteritis. $N$ Engl J Med. 2009;61(18):1776-1785.

6. Lopman B, Zambon M, Brown DW. The Evolution of Norovirus, the "Gastric Flu". PLoS Med. 2010;5(2):187-189.

7. Middleton PJ, Szymanski MT, Petric M. Viruses associated with acute gastroenteritis in young children. Am J Dis Child. 1977;131(7):733-737.

8. American Public Health Association (APHA). Norovirus Infection. In: Heymann David L, editor. Control of Communicable Diseases Manual. 2008. p. 227-229.

9. CDC. Norovirus in Healthcare Facilities Fact Sheet. 2011.

10. Scallan E, Hoekstra RM, Angulo FJ, et al. Foodborne Illness Acquired in the United States-Major Pathogens. Emerg Infect Dis. 2011;17(1):7-15.
11. Patterson T, Hutchings P, Palmer S. Outbreak of SRSV gastroenteritis at an international conference traced to food handled by a post symptomatic caterer. Epidemiol Infect. 1993;111(1):157-162.

12. ExPAsy. SIB Swiss Institute of Bioinformatics/Disclaimer; 2016.

13. Thi Nguyen Hoa-Tran, Toyoko Nakagomi, Daisuke Sano, et al. Department of Hygiene and Molecular Epidemiology, Graduate School of Biomed. Molecular epidemiology of noroviruses detected in Nepalese children with acute diarrhea between 2005 and 2011: Increase and predominance of minor genotype GII 13. 2005.

14. Widdowson MAASW, Bulens SN, Beard RS, et al. Norovirus and foodborne disease, United States, 1991-2000. Emerg Infect Dis. 2005;11:95-102.

15. Mead PS, Slutsker L, Dietz V, et al. Food-related illness and death in the United States. Emerg Infect Dis. 1999;5:607-625.

16. Lopman BA, Reacher MH, Vipond IB, et al. Epidemiology and cost of nosocomialgastroenteritis, Avon, England, 2002-2003. Emerg Infect Dis. 2004;10:1827-1834.

17. The department of Health. Guidelines for the public health management of gastroenteritis outbreaks due to norovirus or suspected viral agents in Australia. 2010 